\title{
Photometric Supernova Typing for the SDSS SN Survey
}

\author{
Elizabeth S. Rivers \\ Wellesley College \\ Office of Science, SULI Program \\ Stanford Linear Accelerator Center \\ Stanford, California
}

August 12, 2005

Prepared in partial fulfillment of the requirements of the Office of Science, U.S, Department of Energy Science Undergraduate Laboratory Internship (SULI) Program under the direction of Dr. Masao Sako of the Kavli Institute for Particle Astrophysics and Cosmology (KIPAC) at the Stanford Linear Accelerator Center (SLAC).

Participant

Signature

Adviser

Signature 


\section{Table of Contents}

$\begin{array}{ll}\text { Abstract } & 3\end{array}$

Introduction 4

$\begin{array}{ll}\text { Methods } & 6\end{array}$

$\begin{array}{ll}\text { Results } & 7\end{array}$

$\begin{array}{ll}\text { Conclusion } & 7\end{array}$

$\begin{array}{ll}\text { Acknowledgments } & 9\end{array}$

$\begin{array}{ll}\text { References } & 9\end{array}$

$\begin{array}{ll}\text { Tables \& Figures } & 10\end{array}$ 


\begin{abstract}
SN Typing for the SDSS SN Survey. Elizabeth S. Rivers (Wellesley College, Wellesley, MA, 02481) Masao Sako (KIPAC/Stanford, Stanford, CA)
\end{abstract}

In the fall of 2004 the Sloan Digital Sky Survey (SDSS) 2.5m telescope scanned the southern equatorial stripe for approximately 20 nights over the space of two months. Light curves for over four dozen supernovae ( $\mathrm{SNe}$ ) were collected over time using five colored filters ugriz that together had a range of approximately $3000 \AA$ to $10500 \AA$. 22 SNe were spectroscopically confirmed with follow-up observation. Using the data obtained in the Fall 2004 campaign, preparations are now being made for the Supernova Survey of the SDSS II, a three-year extension of the original project. One main goal of the Supernova Survey will be to identify and study type Ia SNe of up to redshift $\sim 0.4$, the intermediate 'redshift desert,' as well as enabling further study of other types of SNe including type 1b/c and peculiar SNe. Most of the SNe found will not have spectra taken, due to time and cost constraints. Thus it would be advantageous to be able to robustly type SNe solely from the light curves obtained by the SDSS telescope prior to, or even without ever obtaining a spectrum. Using light curves of well-observed SNe templates were constructed for comparison with unknown SNe in order to photometrically type them. 
Red and bloated spheres

Explode like hot tomatoes.

I'm watching stars die

\section{Introduction}

\section{SUPERNOVA CLASSIFICATION}

Supernovae (SNe) come in a great variety, ranging in magnitude, redshift and light curve shape. Due in main part to the history of SN discovery, classification is typically based on optical spectra taken of the SNe near maximum light [1]. By definition type II SNe show strong hydrogen emission lines, absent in type I SNe. The types are further broken down into several subtypes - Ia, the famous standard candles that allow astronomers to measure distances to distant galaxies, distinguished by Si II lines in their spectra; Ib which shows no Si II, and Ic, with no Si II and also no helium. Type II are sometimes subclassified based on the profile of the hydrogen line (IIn showing a narrow $\mathrm{H}$ line) as well as on their light curveshapes.

In reality $\mathrm{Ib} / \mathrm{c}$ SNe share more in common with type II SNe than they do with Ia. Both type II and type Ib/c SNe mark the deaths of massive stars. When a star reaches the end of its life of hydrogen burning there are a few paths it may take depending on the mass of the star in question. If it is a relatively light star, around three solar masses or less, then it goes out with the proverbial whimper, collapsing down into a white dwarf with little to mark its passing. If the star is a bit heavier, then it becomes a red giant, fusing hydrogen, helium and heavier elements into iron. Once the giant has fused all that it is able, it dies in a rather complicated manner - cooling, contracting and then blowing its outer layers outward in a massive explosion that leaves behind the collapsed core in the form of a neutron star or a black hole. It is theorized that type $\mathrm{Ib} / \mathrm{c}$ SNe show differences in their spectra due to the loss of their outer atmospheres prior to going nova.

The mechanism behind a type Ia SN is quite different from that of the core-collapse SNe 
and is unique in the degree of homogeoneity it gives to Ia light curves. It is believed that a type Ia SN is caused by a white dwarf, essentially a small, dead star, becoming heavy enough that the electron-degeneracy pressure keeping it up can no longer balance the gravitational force pulling it in and the star collapses into a neutron star, expelling its atmosphere in a massive SN. This process however only occurs when a white dwarf grows to a certain size by accreting mass from a larger star in a binary system. Since this occurs at the same size for any white dwarf, type Ia SNe are necessarily homogeneous, showing very nearly identical light curves from one occurrence to another and virtually always peaking at the same magnitude of brightness. Consequently they make excellent standard candles - that is since their absolute magnitude is already known, their distance can be calculated from their apparent magnitude in the sky. It might be expected that all type Ia SNe are exactly identical. This, however, is not the case. Some type Ia's are brighter than others and some have peculiarly shaped light curves. It is still possible to determine absolute magnitude from algorithms using the decay rate of the light curve, but further study is required to understand the causes of the observed diversity.

\section{THE SLOAN DIGITAL SKY SURVEY}

The Sloan Digital Sky Survey (SDSS) II will begin observing in the fall of 2005 and will survey the southern equatorial stripe for periods of three months in the falls of 2005 - 2007. It will cover 350 square degrees of sky, taking data for each section every other observing night. The Supernova Survey will be a major subcomponent of this study, and its primary goal is to obtain high-quality, multi-color light curves of $\sim 200$ Ia SNe in the intermediate redshift interval of $\mathrm{z}=0.05-0.35$ [2]. The survey will provide multi-band light curves measured with the SDSS 2.5m telescope at Apache Point Observatory. The SDSS focal plane camera is equipped with 5 filters ugriz [figure] that together cover a wavelength range of approximately $3000 \AA$ to $10500 \AA$.

During the first Supernova Survey light curves were inspected manually, comparing the curves with standard models of the homogeneous type Ia looking for matches. Spectra were 
taken of $22 \mathrm{SN}$ candidates that were suspected of being type Ia and of these 16 SNe were spectroscopically confirmed to be Ia. The rest were were found to be 1 type Ib/c and 5 type II SNe. Our goal is to improve upon this classification of SNe observed by the SDSS telescope for the upcoming survey.

\section{Methods}

One way to type SNe lacking spectra is to compare the measured light curves with those of other known spectroscopically confirmed SNe. This is, however, not very straightforward, since the light curves of most SNe in the literature have been measured in the Johnson/Cousins filter set $U B V R I$, which have filter transmission curves that differ quite markedly from those of the SDSS filters. Transformation of magnitudes in one filter set to another is not easy to do unless you know the underlying spectrum well. This is also problematic when comparing SNe at different cosmological redshifts, since strong spectral features may shift in and out of filter band passes.

Additionally, the diversity of SNe makes it difficult to create a truly standard model for any class, with the possible exception of type Ia. SNe resulting from the core-collapse of a giant stars tend to show a wide range of properties, particularly in the many different light curveshapes they exhibit. Type II SNe are sometimes broken down into subtypes based on light curveshape: type IIP and type IIL, 'P' for the $~ 100$ day plateau following peak brightness and 'L' for the linear decay of IIL light curves.

We choose to construct a library of multi-epoch template spectra in the SDSS filters and calculated on a grid of redshift $z$ up to 0.4 and extinction $A_{V}$ up to 0.5 . This library is built of template spectra from P. Nugent (UC Berkely, CA) and $U B V I R$ light curves and spectra from [4] transformed into the SDSS filter set. Each light curve observed by SDSS can now be compared with the templates, shifted on the grid of $z$ and $A_{V}$, fitting the data to each

model curve. The best fit (lowest $\chi^{2}$ ) template is chosen as the most likely SN type. If and when new SN are uncovered with sufficient photometric and spectral data they can be added 
to the library for improved accuracy in typing.

\section{Results}

Using the ugri filters we calculate the $\chi^{2}$ for the SNe light curvein each filter [Figures 1\&2].

We can then select the lowest $\chi^{2}$ value as the most likely SN type using 3,6 or 10 epochs. Applying this to the 22 confirmed SNe we find that 20/22 are typed correctly with maximum number of epochs [Table 1] and 19/22 are typed correctly with only 3 epochs.

We also used our photometric method to type nearly 60 SNe lacking spectra, finding 40 Ia, 5 Ibc and 10 type II SNe. 23 type Ia were confidently typed using all available epochs [Table 2] and a number of these will recieve follow-up work.

\section{Conclusion}

For the 22 spectroscopically confirmed SNe from the initial run of the SDSS Survey we are able to type with $\sim 90 \%$ accuracy. Given only 22 test cases, each with good data, the percent-accuracy is likely to change when the method is applied to large numbers of SNe.

Of the 58 unconfirmed SN candidates that we attempted to type, three were completely ambiguous and several jumped suddenly in $\chi^{2}$ from 6 to 10 epochs, adding doubt to the confidence of their typing. Additionally, none of the confirmed SNe was stamped with a Ibc type, so it is difficult to say what kind of accuracy we should really expect out of the five unconfirmed SNe that recieved this type. However several of the unconfirmed SNe appear to have been well-typed. We plan to obtain spectra of host galaxies of a few of these SN Ia candidates that have good photometric data. If the host galaxy spectroscopic redshift is consistent with the redshift estimated from the light curves, we will include them in the sample of confirmed SNe.

Given the volume of the SDSS and the expense involved with taking spectra, it would seem best to implement additional parameters when choosing Ia candidates for spectral 
analysis. Placing an upper limit on the $\chi^{2}$ value, a lower limit on the distance between the best and second-best fit light curves and limiting our candidates to the ones with the best photometry (in this case, smoothest curve and smallest error-bars) should greatly increase our chances of actually sifting out the best Ia Supernovae for study. 


\section{Acknowledgments}

The US. Dept. of Energy, Office of Science for opportunity and funding. Masao and Phil for excellent mentoring, Peter Nugent whom I have never met, My father for his lovely tech support, Lowry, Vidya and the people in the office next door for all the rest.

Thanks guys.

\section{References}

[1] Filipppenko A. F. "Optical Spectra of SNe." Annu. Rev. Astron. Astrophys. 1997.

[2] Sako, M. et al. "The Fall 2004 SDSS SN Survey." Dec, 2004. 22nd Texas Symposium on Relativistic Astrophysics, Stanford, CA, 2004.

[3] The Online Supernova Spectrum Website. [Online Database] Available HTTP:

http://bruford.nhn.ou.edu/ suspect/index1.html 


\section{Tables \& Figures}

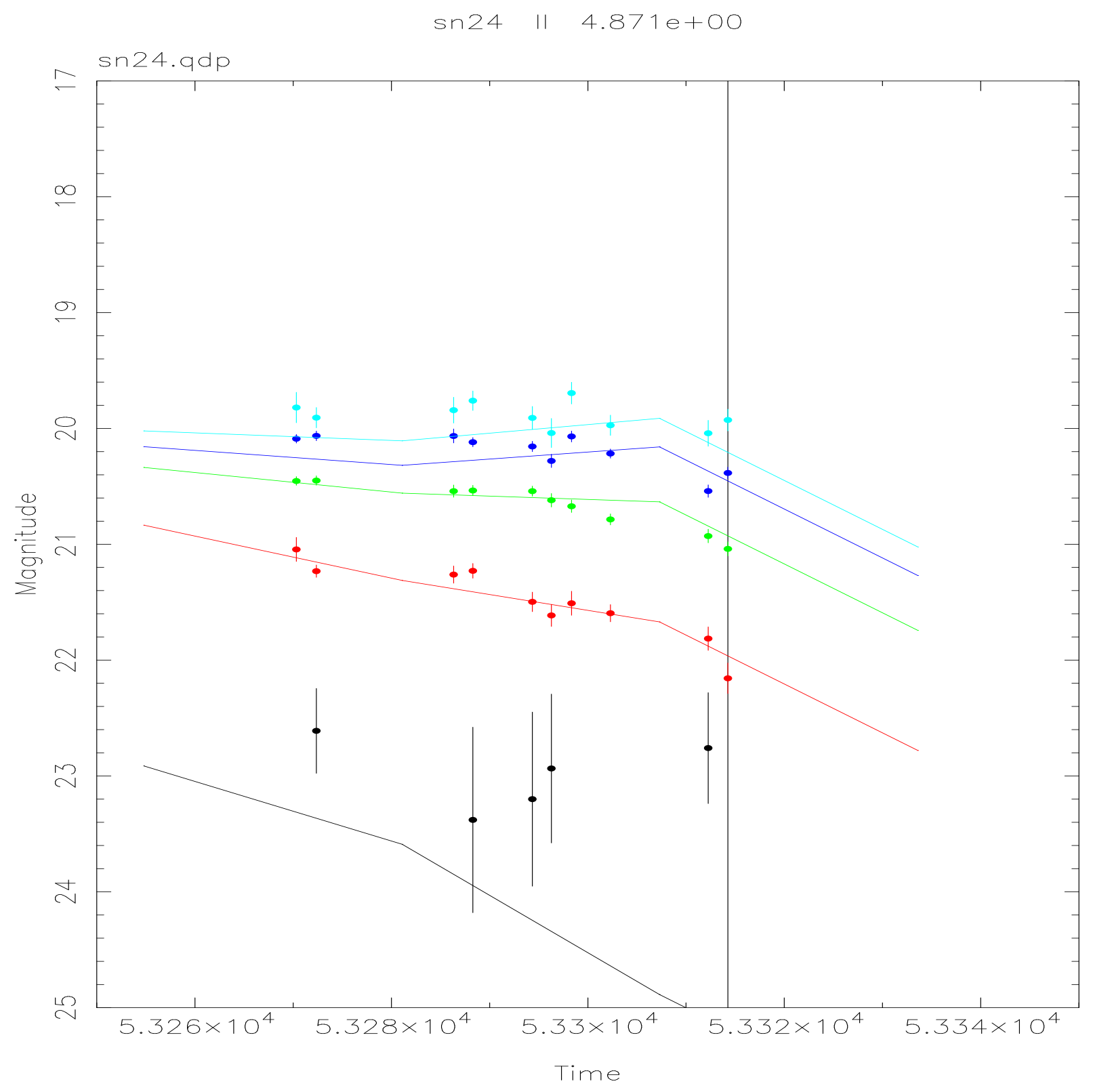




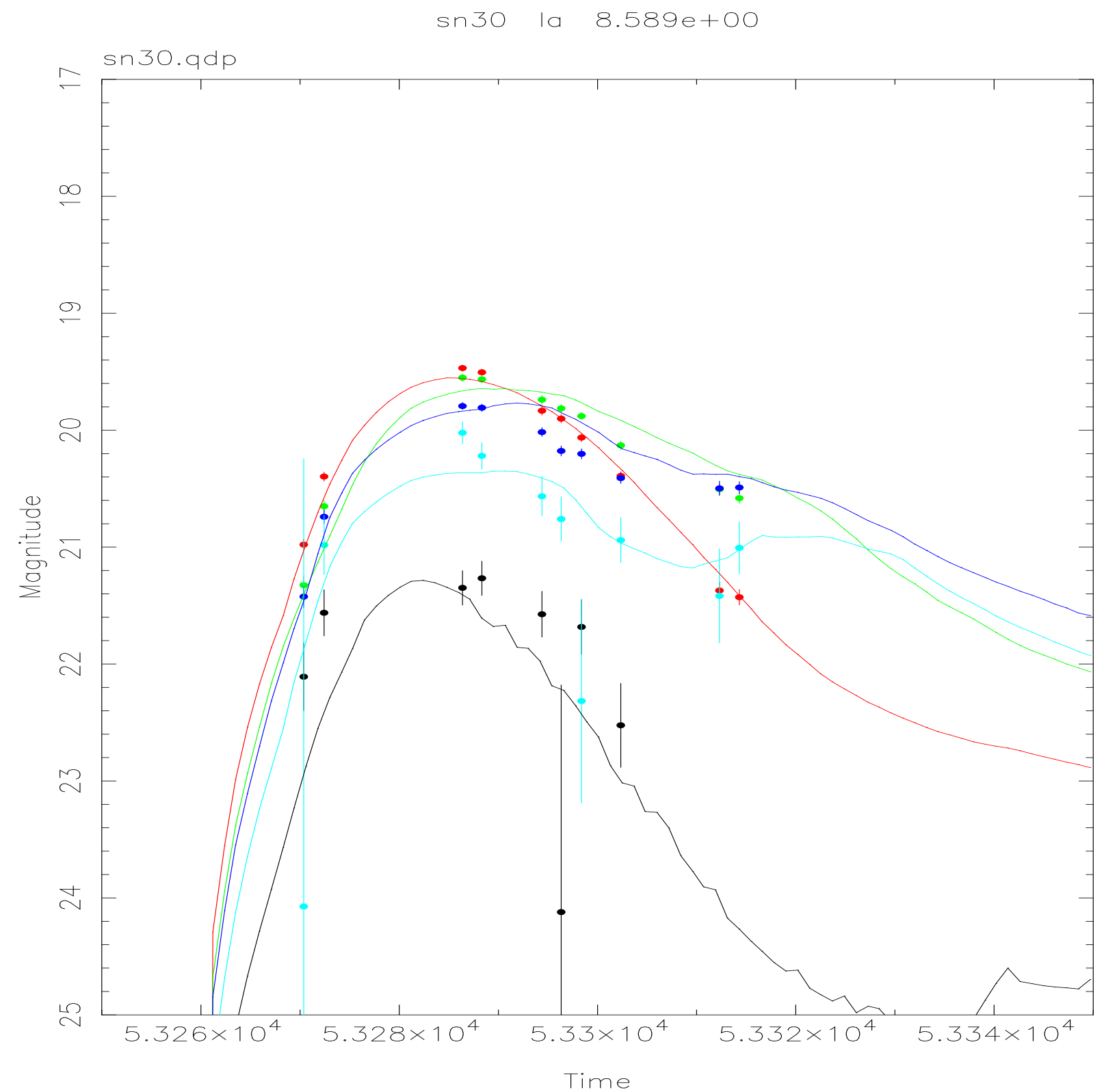


Table 1: Confirmed Supernovae

\begin{tabular}{|c||c|c|c|}
\hline SN & Type & Best-Fit Type & Best-Fit $\chi^{2}$ (max epochs) \\
\hline \hline $\mathbf{5}$ & Ia & Ia & $1.231 \mathrm{e}+01$ \\
$\mathbf{9}$ & II & II & $2.960 \mathrm{e}+01$ \\
$\mathbf{1 0}$ & Ia & Ia & $5.893 \mathrm{e}+00$ \\
$\mathbf{1 2}$ & II & II & $1.304 \mathrm{e}+01$ \\
$\mathbf{1 5}$ & Ia & Ia & $1.819 \mathrm{e}+01$ \\
$\mathbf{1 8}$ & II & Ia & $1.111 \mathrm{e}+02$ \\
$\mathbf{1 9}$ & Ia & Ia & $4.586 \mathrm{e}+01$ \\
$\mathbf{2 0}$ & Ib & Ia & $1.641 \mathrm{e}+01$ \\
$\mathbf{2 4}$ & II & II & $4.871 \mathrm{e}+00$ \\
$\mathbf{2 5}$ & Ia & Ia & $5.316 \mathrm{e}+00$ \\
$\mathbf{3 0}$ & Ia & Ia & $8.589 \mathrm{e}+00$ \\
$\mathbf{8 3}$ & Ia & Ia & $2.403 \mathrm{e}+01$ \\
$\mathbf{1 1 1}$ & II & II & $1.211 \mathrm{e}+01$ \\
$\mathbf{1 2 8}$ & Ia & Ia & $8.701 \mathrm{e}+01$ \\
$\mathbf{1 3 3}$ & Ia & Ia & $4.608 \mathrm{e}+01$ \\
$\mathbf{1 7 1}$ & Ia & Ia & $1.207 \mathrm{e}+02$ \\
$\mathbf{1 7 2}$ & Ia & Ia & $3.537 \mathrm{e}+01$ \\
$\mathbf{1 7 6}$ & Ia & Ia & $7.317 \mathrm{e}+01$ \\
$\mathbf{1 9 1}$ & Ia & Ia & $4.372 \mathrm{e}+01$ \\
$\mathbf{1 9 4}$ & Ia & Ia & $4.708 \mathrm{e}+01$ \\
$\mathbf{2 4 2}$ & Ia & Ia & $1.922 \mathrm{e}+01$ \\
$\mathbf{2 4 7}$ & Ia & Ia & $3.334 \mathrm{e}+00$ \\
\hline
\end{tabular}


Table 2: Unconfirmed Supernovae: Well-Typed Ia Supernovae

\begin{tabular}{|c|c||c|c|}
\hline SN & Best-Fit $\chi^{2}($ max epochs $)$ & SN & Best-Fit $\chi^{2}$ (max epochs) \\
\hline \hline $\mathbf{1 0 8}$ & $8.591 \mathrm{e}-02$ & $\mathbf{3 5}$ & $3.605 \mathrm{e}+00$ \\
$\mathbf{1 0 0}$ & $1.309 \mathrm{e}+00$ & $\mathbf{1 2 3}$ & $4.112 \mathrm{e}+00$ \\
$\mathbf{9 3}$ & $1.693 \mathrm{e}+00$ & $\mathbf{9 6}$ & $4.658 \mathrm{e}+00$ \\
$\mathbf{1 1 7}$ & $1.698 \mathrm{e}+00$ & $\mathbf{3 2}$ & $5.023 \mathrm{e}+00$ \\
$\mathbf{4}$ & $2.343 \mathrm{e}+00$ & $\mathbf{9 4}$ & $5.209 \mathrm{e}+00$ \\
$\mathbf{3 3}$ & $2.446 \mathrm{e}+00$ & $\mathbf{5 1}$ & $5.669 \mathrm{e}+00$ \\
$\mathbf{2 8}$ & $2.869 \mathrm{e}+00$ & $\mathbf{3 7}$ & $5.760 \mathrm{e}+00$ \\
$\mathbf{1 3 0}$ & $3.057 \mathrm{e}+00$ & $\mathbf{4 1}$ & $6.334 \mathrm{e}+00$ \\
$\mathbf{1 1 6}$ & $3.070 \mathrm{e}+00$ & $\mathbf{9 1}$ & $6.349 \mathrm{e}+00$ \\
$\mathbf{8 8}$ & $3.366 \mathrm{e}+00$ & $\mathbf{8 6}$ & $8.398 \mathrm{e}+00$ \\
$\mathbf{1 2 5}$ & $3.429 \mathrm{e}+00$ & $\mathbf{2 9}$ & $8.622 \mathrm{e}+00$ \\
$\mathbf{1 1 0}$ & $3.453 \mathrm{e}+00$ & & \\
\hline
\end{tabular}

